\title{
A Novel Coaxial Magnetic Gear Using Bulk HTS for Industrial Applications
}

\author{
Linni Jian, Student Member, IEEE, K. T. Chau, Senior Member, IEEE, Wenlong Li, Student Member, IEEE, and \\ Jiangui Li, Student Member, IEEE
}

\begin{abstract}
In this paper, a novel coaxial magnetic gear (CMG) using bulk high temperature superconductors (HTS) is proposed. By substituting bulk HTS for ferromagnetic material, a novel stationary ring is resulted, which can not only provide the desired field modulation effect but also suppress the end-effects that commonly occurred in the traditional CMG. The performance of the proposed bulk HTS CMG is assessed and then verified by using finite element analysis.
\end{abstract}

Index Terms-Coaxial magnetic gear, field modulation, high temperature superconductor, permanent magnet, torque transmission.

\section{INTRODUCTION}

G EARS have been extensively used in many industrial applications for transmitting torques and adjusting speeds. So far, mechanical gears are still the leading players in this role. However, because of their contact mechanism, the associated transmission loss, noise, vibration, and thus regular lubrication are inevitable. With the advent of high-energy permanent magnets (PMs), the concept of non-contact torque transmission through the interaction between PMs has attracted increasing attention. Consequently, a PM based gear is so-called the magnetic gear (MG).

The early MGs are merely simple analogs of the mechanical ones [1]. Torque transmission is achieved by the direct interaction of closely arrayed PMs. The biggest shortcoming of such MGs is their poor torque densities. Although several attempts have been conducted for improving their torque density, such as optimizing the design parameters [2] and adopting bulk high temperature superconductors (HTS) to provide stronger magnetic field [3], they are still far away from satisfying the demands of industrial applications. Recently, a novel type of MGs using the field modulation effect has been proposed [4]-[6]. It adopts a coaxial topology, so-called the coaxial MG (CMG), which can simultaneously engage all the PMs for torque transmission. Thus, its torque density can be dramatically improved. Moreover, the coaxial topology makes it readily be integrated with electric machinery to meet the demands arising from wind power generation [7] or electric vehicles [8].

Manuscript received October 18, 2009. First published March 11, 2010; current version published May 28, 2010. This work was supported by a grant (HKU 7105/07E) from the Research Grants Council, Hong Kong Special Administrative Region, China.

The authors are with the Department of Electrical and Electronic Engineering, The University of Hong Kong, Hong Kong. (e-mail: lnjian@eee.hku.hk).

Color versions of one or more of the figures in this paper are available online at http://ieeexplore.ieee.org.

Digital Object Identifier 10.1109/TASC.2010.2040609

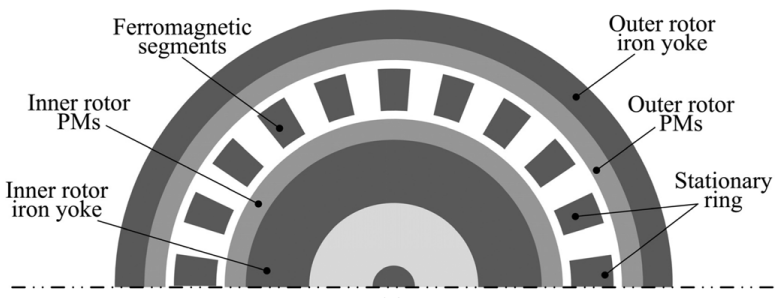

(a)

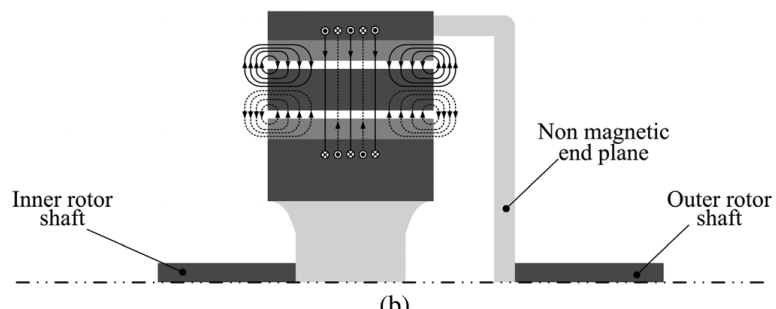

(b)

Fig. 1. CMG topology (upper half). (a) Front view. (b) Side view.

Fig. 1 shows the topology (upper half) of the CMG. It consists of three main parts: the inner rotor, the stationary ring and the outer rotor. PMs are mounted on the surfaces of the two rotors. The stationary ring consists of ferromagnetic segments and air slots. Once the number of the ferromagnetic segments $N_{s}$ equals the sum of the pole-pair number of the inner rotor $p_{1}$ and the pole-pair number of the outer rotor $p_{2}$, stable torque transmission between the two rotors can be achieved, and the gear ratio of $-p_{1}: p_{2}$ is resulted. Herein, the minus notation means opposite directions of two rotors. The modulation effect induced by the stationary ring is essential to the torque transmission and speed variation. In order to realize satisfactory field modulation, the ferromagnetic segments have to be thick enough in the radial direction. Also, because of high permeability of the ferromagnetic segments along the axial direction, there is a lot of leakage flux occurring at the end-spaces as depicted in Fig. 1(b). Such serious end-effects will result in 30\% reduction of the torque density of CMGs [9].

The purpose of this paper is to propose a novel CMG in which bulk HTS are substituted for ferromagnetic segments to provide the desired modulation effect and to suppress the undesirable end-effects. In Section II, the modulation effect will be analyzed. In Section III, the ideal of field modulation using HTS will be introduced. The performance analysis of the proposed bulk HTS CMG will be presented in Section IV. Finally, the conclusion is drawn in Section V.

\section{Modulation Effect of Stationary Ring}

For simplicity, the permeability of the iron yokes is assumed to be infinite, and the permeability of the PMs is assumed to 


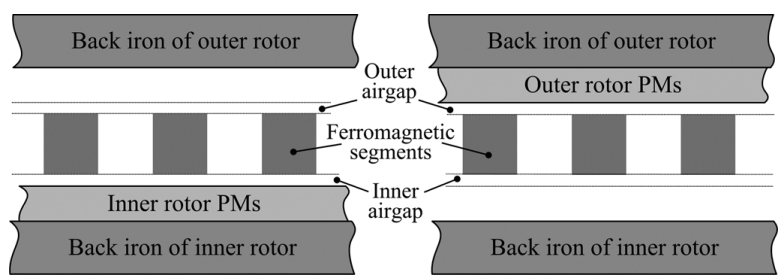

Fig. 2. Field superposition in CMG.
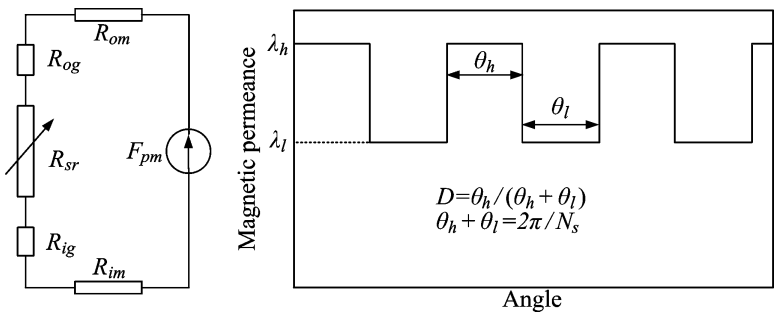

Fig. 3. Equivalent magnetic circuit and magnetic permeance waveform of 1-D path model.

be that of the air space. Since the nonlinear factors are absent, the magnetic field distribution in the CMG can be considered as superposition of the fields excited by individual rotors. Thus, the CMG can be represented in pseudo-polar coordinates as shown in Fig. 2.

When the 1-D path model is adopted, the circumferential component of magnetic field can be ignored. Fig. 3 shows the equivalent magnetic circuit. Thus, the magnetic flux density excited by the inner rotor PMs $B_{i p m}$ and that excited by the outer rotor PMs $B_{o p m}$ can be expressed as:

$$
\begin{aligned}
B_{i p m} & =F_{i p m}(\theta) \cdot \Lambda(\theta) \\
B_{\text {opm }} & =F_{\text {opm }}(\theta) \cdot \Lambda(\theta)
\end{aligned}
$$

where $F_{i p m}$ and $F_{o p m}$ are the magnetic motive forces (MMFs) provided by the inner rotor PMs and the outer rotor PMs, respectively, and $\Lambda$ is the magnetic permeance in radial direction. Without considering the high-order harmonics, the MMFs can be written in the form of fundamental components:

$$
\begin{aligned}
& F_{i p m}(\theta)=\frac{B_{i r} h_{i p m}}{\mu_{0}} \cos p_{i}\left(\theta+\omega_{i} t+\theta_{0}^{i}\right) \\
& F_{o p m}(\theta)=\frac{B_{o r} h_{o p m}}{\mu_{0}} \cos p_{o}\left(\theta+\omega_{o} t+\theta_{0}^{o}\right)
\end{aligned}
$$

where $B_{i r}$ and $B_{o r}$ are the remanences of the inner rotor PMs and the outer rotor PMs, respectively, $p_{i}$ and $p_{o}$ are the polepair numbers of the inner rotor PMs and the outer rotor PMs, respectively, $\omega_{i}$ and $\omega_{o}$ are the rotational speeds of the two rotor, $\theta_{0}^{i}$ and $\theta_{0}^{o}$ are the initial phase angles of the two rotors, $h_{i p m}$ and $h_{\text {opm }}$ are the heights of the inner rotor PMs and the outer rotor PMs, respectively. Also, the magnetic permeance can be expressed as:

$$
\Lambda(\theta)=\frac{1}{R_{i m}+R_{o m}+R_{i g}+R_{o g}+R_{s r}(\theta)}
$$

where $R_{i m}=h_{i p m} / \mu_{0}, R_{o m}=h_{o p m} / \mu_{0}, R_{i g}=h_{i g} / \mu_{0}$ and $R_{o g}=h_{o g} / \mu_{0}$ are the magnetic reluctances in radial direction of the inner rotor PMs, the outer rotor PMs, the inner airgap and the outer airgap, respectively, $R_{s r}(\theta)=h_{s r} / \mu_{0}$ or 0 is the



Fig. 4. Modulation effect of stationary ring versus shape factors of ferromagnetic segment.

magnetic reluctance in radial direction of the stationary ring, $h_{i g}, h_{o g}$ and $h_{s r}$ are the height of the inner airgap, the outer airgap and the stationary ring, respectively.

Consequently, the waveform of the magnetic permeance in radial direction is shown in Fig. 3. It can be expanded in form of Fourier series:

$$
\Lambda(\theta)=\lambda_{0}+\lambda_{1} \cos N_{s} \theta+\sum_{j=2}^{+\infty} \lambda_{j} \cos j N_{s} \theta
$$

where $N_{s}$ is the number of ferromagnetic segments. The magnitudes of the zeroth-order and the first-order components are $\lambda_{0}=2(1-D) \lambda_{l}+2 D \lambda_{h}$ and $\lambda_{1}=2\left(\lambda_{h}-\lambda_{l}\right) \sin (D \pi) / \pi$. Herein, $\lambda_{h}=\mu_{0} / H, \lambda_{l}=\mu_{0} /\left(H+h_{s r}\right), H=h_{\text {ipm }}+h_{\text {opm }}+$ $h_{i g}+h_{o g}, D=\theta_{h} /\left(\theta_{h}+\theta_{l}\right), \theta_{h}$ and $\theta_{l}$ are the widths of the ferromagnetic segment and the air slot, respectively, as shown in Fig. 3(b).

From (1)-(4) and (6), the zeroth-order and the first-order harmonics of the magnetic flux density excited by the inner rotor PMs and the outer rotor PMs can be obtained as:

$$
\begin{aligned}
B_{i p m}^{0}= & \frac{\lambda_{0} B_{i r} h_{i p m}}{\mu_{0}} \cos p_{i}\left(\theta+\omega_{i} t+\theta_{0}^{i}\right) \\
B_{i p m}^{1}= & \frac{\lambda_{1} B_{i r} h_{i p m}}{\mu_{0}} \cos p_{i}\left(\theta+\omega_{i} t+\theta_{0}^{i}\right) \cos N_{s} \theta \\
= & \frac{\lambda_{1} B_{i r} h_{i p m} \cos \left(N_{s}+p_{i}\right)\left(\theta+\frac{p_{i} \omega_{i} t}{N_{s}+p_{i}}+\frac{p_{i} \theta_{0}^{i}}{N_{s}+p_{i}}\right)}{2 \mu_{0}} \\
& +\frac{\lambda_{1} B_{i r} h_{i p m} \cos p_{o}\left(\theta+\omega_{o} t+\frac{p_{i} \theta_{0}^{i}}{p_{o}}\right)}{2 \mu_{0}} \\
= & B_{i p m}^{11}+B_{i p m}^{12} \\
B_{o p m}^{0}= & \frac{\lambda_{0} B_{o r} h_{o p m}}{\mu_{0}} \cos p_{o}\left(\theta+\omega_{o} t+\theta_{0}^{o}\right) \\
B_{o p m}^{1}= & \frac{\lambda_{1} B_{o r} h_{o p m}}{\mu_{0}} \cos p_{o}\left(\theta+\omega_{o} t+\theta_{0}^{o}\right) \cos N_{s} \theta \\
= & \frac{\lambda_{1} B_{o r} h_{o p m} \cos \left(N_{s}+p_{o}\right)\left(\theta+\frac{p_{o} \omega_{o} t}{N_{s}+p_{o}}+\frac{p_{o} \theta_{0}^{o}}{N_{s}+p_{o}}\right)}{2 \mu_{0}} \\
& +\frac{\lambda_{1} B_{o r} h_{o p m} \cos p_{i}\left(\theta+\omega_{i} t+\frac{p_{o} \theta_{0}^{o}}{p_{i}}\right)}{2 \mu_{0}} \\
= & B_{o p m}^{11}+B_{o p m}^{12}
\end{aligned}
$$

It can be found that the components $B_{i p m}^{0}$ and $B_{o p m}^{12}$ have the same pole-pair number and the rotational speed. Thus, they can interact with each other to develop stable magnetic torque 


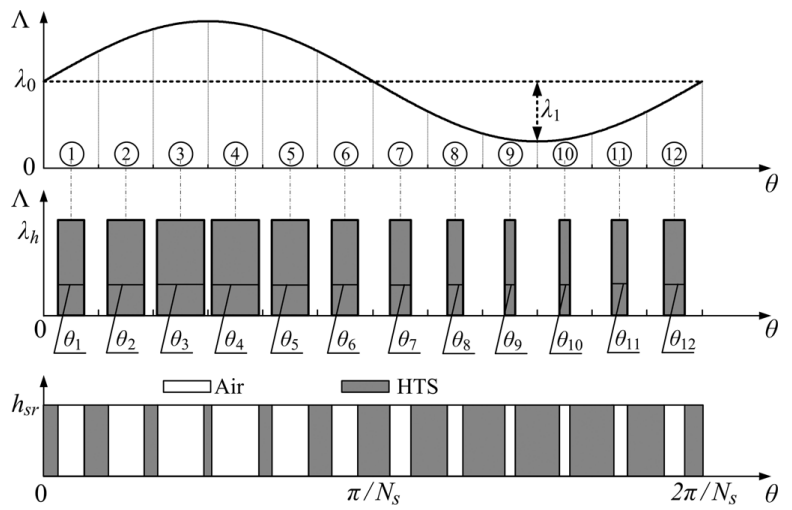

Fig. 5. Field modulation using PWM.

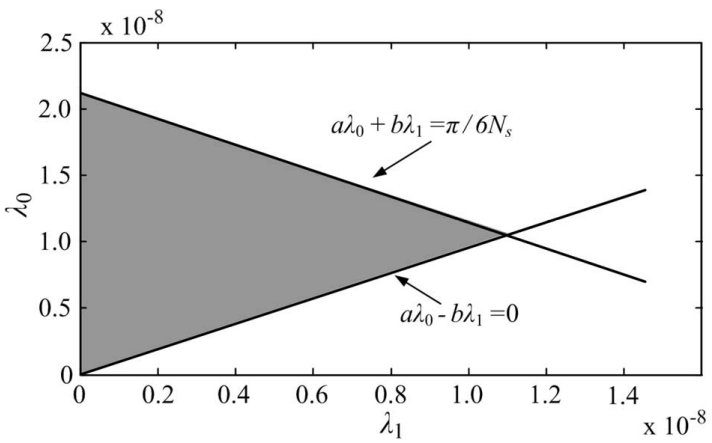

(a)

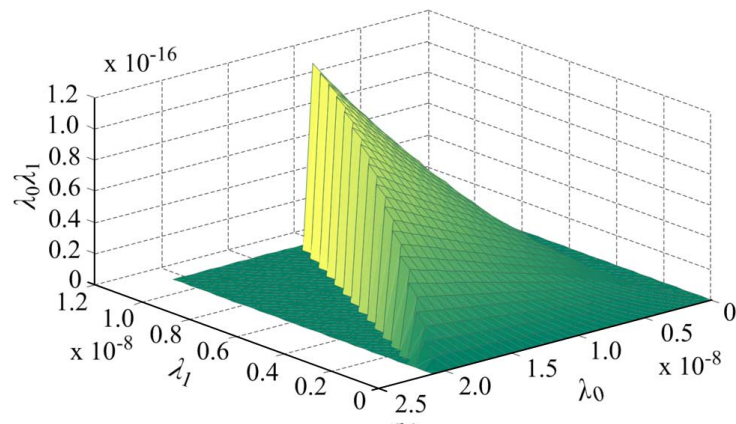

(b)

Fig. 6. Maximization of field modulation. (a) Selection region. (b) Modulation effect.

$T_{i}$ on the inner rotor. In addition, the components $B_{o p m}^{0}$ and $B_{i p m}^{12}$ have the same pole-pair number and the rotational speed. Thus, they can interact with each other to develop stable magnetic torque $T_{o}$ on the outer rotor. Hence, they can be expressed as:

$$
\begin{aligned}
& T_{i}=\frac{s p_{i} B_{i r} B_{o r} h_{o p m} h_{i p m} \lambda_{0} \lambda_{1}}{2 \mu_{0}^{2}} \cos \left(p_{i} \theta_{0}^{i}-p_{o} \theta_{0}^{o}\right) \\
& T_{o}=\frac{s p_{o} B_{i r} B_{o r} h_{o p m} h_{i p m} \lambda_{0} \lambda_{1}}{2 \mu_{0}^{2}} \cos \left(p_{i} \theta_{0}^{i}-p_{o} \theta_{0}^{o}\right)
\end{aligned}
$$

where $s$ is the circumferential length.

It can be seen that besides the term $s B_{i r} B_{o r} h_{o p m} h_{i p m}$ which is corresponding to the magnetic intensity provided by the PMs, the term $\lambda_{0} \lambda_{1}$ which represents the modulation effect of the stationary ring also decides the torque transmission capacity of the CMG. Fig. 4 shows the relationship between the modulation effect and shape factors of the ferromagnetic segments. It can

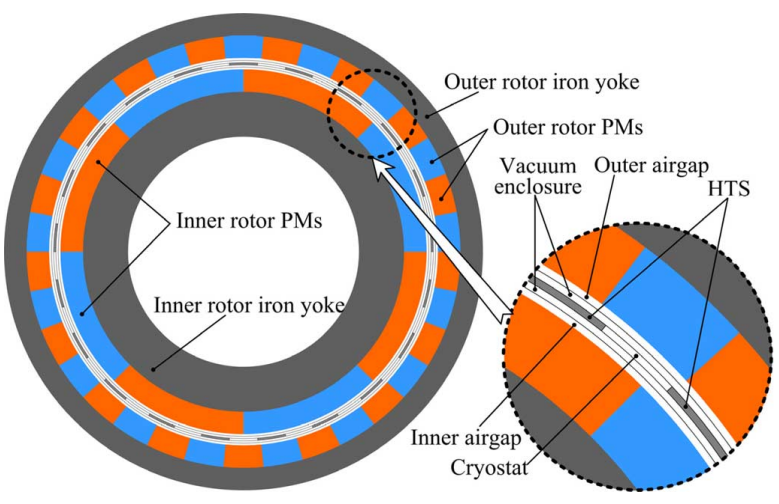

Fig. 7. Proposed CMG topology.

be found that in order to obtain the satisfactory modulation effect, the width of the air slot should be equal to the width of the ferromagnetic segment. Most importantly, the height of the stationary ring should be 3-4 times the total height of the PMs and the airgaps. On the one hand, this will increase the volume of the CMG; on the other hand, this inevitably arouses the serious end-effects and deteriorates the torque transmission capacity.

\section{Field Modulation Using Bulk HTS}

In order to overcome the shortcomings caused by the ferromagnetic segments, bulk HTS are introduced to offer a novel field modulation. According to the Meissner effect, HTS working below the critical temperature $T_{c}$ rejects magnetic field penetration. When the air slots in the traditional stationary ring are filled with HTS, the magnetic permeance towards HTS is virtually zero. Since these bulk HTS are independent of the height of the stationary ring, the HTS stationary ring can be fabricated with a very short length in radial direction. Thus, the ferromagnetic material can be totally abandoned.

The high-order harmonics given in (6) have no contribution to the torque transmission but arouse torque ripples [10]. In order to reduce high-order harmonics, as shown in Fig. 5, the pulse wide modulation (PWM) is adopted in the field modulation. The pulse widths are given by:

$$
\begin{aligned}
\lambda_{h} \theta_{i} & =\int_{(i-1) \pi / 6 N_{s}}^{i \pi / 6 N_{s}}\left(\lambda_{0}+\lambda_{1} \sin N_{s} \theta\right) d \theta \\
\theta_{i} & =\left(\frac{\pi}{6 N_{s} \lambda_{h}}\right) \lambda_{0}+\left(\frac{2}{N_{s} \lambda_{h}} \sin \frac{\pi}{12} \sin \frac{(2 i-1) \pi}{12}\right) \lambda_{1}
\end{aligned}
$$

In order to maximize the modulation effect $\lambda_{0} \lambda_{1}$, the following constraints are deduced:

$$
\begin{aligned}
& \theta_{3}=a \lambda_{0}+b \lambda_{1} \leq \frac{\pi}{6 N_{s}} \\
& \theta_{9}=a \lambda_{0}-b \lambda_{1} \geq 0
\end{aligned}
$$

where $a=\pi /\left(6 N_{s} \lambda_{h}\right), b=2 \sin (\pi / 12) \sin (5 \pi / 12) /\left(N_{s} \lambda_{h}\right)$.

Fig. 6(a) gives the selection region of $\lambda_{0}$ and $\lambda_{1}$. The corresponding modulation effect $\lambda_{0} \lambda_{1}$ is depicted in Fig. 6(b). It can be observed that when $\lambda_{0}=\pi /\left(12 a N_{s}\right)$ and $\lambda_{1}=\pi /\left(12 b N_{s}\right)$, the maximum value of $\lambda_{0} \lambda_{1}$ can be attained, hence maximizing the field modulation effect. 
TABLE I

SPECIFICATIONS OF PROPOSED CMG

\begin{tabular}{ll}
\hline \hline Inside radius of inner rotor yoke $[\mathrm{mm}]$ & 51.2 \\
Thickness of PMs on both rotors [mm] & 10 \\
Thickness of stationary ring [mm] & 3 \\
Outside radius of outer rotor yoke [mm] & 106.2 \\
Axial length [mm] & 80 \\
Remanence of PMs [T] & 1.3 \\
\hline \hline
\end{tabular}



$2.00 \mathrm{e}+00$

$1.78 \mathrm{e}+00$

$1.56 \mathrm{e}+00$

$1.33 \mathrm{e}+00$

$1.11 \mathrm{e}+00$

$8.89 \mathrm{e}-01$

$6.67 \mathrm{e}-01$

$4.44 \mathrm{e}-01$

$2.22 \mathrm{e}-01$

$0.00 \mathrm{e}+00$

Unit: Tesla (T)

Fig. 8. Magnetic flux distribution in proposed CMG.

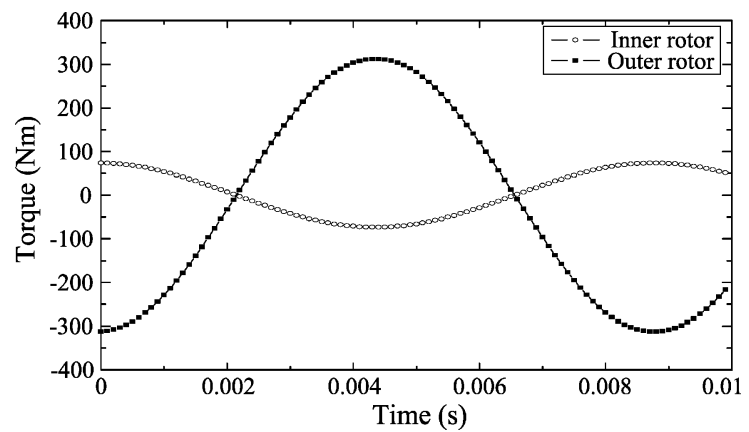

Fig. 9. Torque transmission capacity.

\section{Performance AnAlysis}

Although the proposed PWM field modulation can achieve the minimum torque ripples, the complexity of fabrication is significantly increased. Thus, taking into account the feasibility for practical applications, the topology of the proposed CMG is shown in Fig. 7. The pole-pair numbers of the inner rotor PM and the outer rotor PM are 4 and 17, respectively. There are 21 bulk HTS symmetrically arranged in the stationary ring to offer the desired field modulation effect. The specifications are listed in Table I.

Fig. 8 shows the magnetic field distribution in the proposed CMG calculated by using the finite element analysis (FEA). When the outer rotor is kept still and the inner rotor is rotating at $1700 \mathrm{rpm}$, the calculated waveforms of torques developed on the two rotors are illustrated in Fig. 9. It can be seen that the pull-out

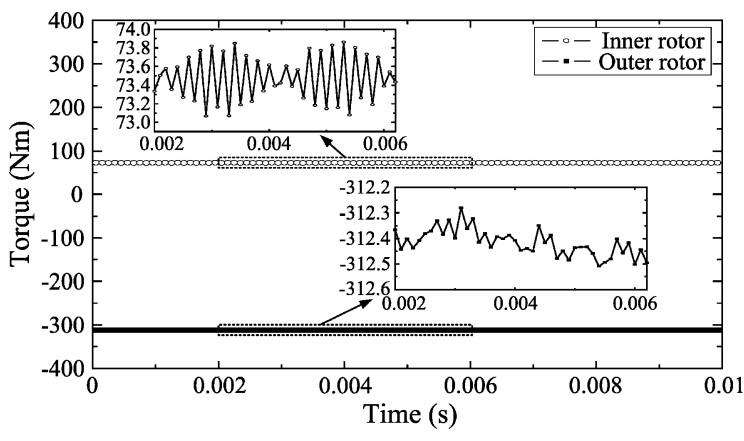

Fig. 10. Stable torque transmission.

torques on the inner rotor and the outer rotor are $312 \mathrm{Nm}$ and $73.4 \mathrm{Nm}$, respectively. Considering the volume sizes listed in Table I, the calculated torque density is $110 \mathrm{kNm} / \mathrm{m}^{3}$. When the inner rotor and the outer rotor are rotating at $1700 \mathrm{rpm}$ and $-400 \mathrm{rpm}$, respectively, the calculated waveforms of torques developed on the two rotors are shown in Fig. 10. It demonstrates that stable torque transmission can be achieved. Also, the corresponding torque ripples are very acceptable, even in the absence of using PWM for field modulation.

\section{CONCLUSION}

In this paper, a novel bulk HTS CMG has been designed and analyzed. By using the 1-D path model, the modulation effect induced by the stationary ring is analyzed. Hence, the introduction of bulk HTS not only provides the desired field modulation effect but also suppresses the undesirable end-effects. Finally, the performance of the proposed CMG is assessed and then verified by using the FEA.

\section{REFERENCES}

[1] E. P. Furlani, "A two-dimensional analysis for the coupling of magnetic gears," IEEE Trans. Magn., vol. 33, no. 3, pp. 2317-2321, May 1997.

[2] S. Kikuchi and K. Tsurumoto, "Trial construction of a new magnetic skew gear using permanent magnet," IEEE Trans. Magn., vol. 30, no. 6, pp. 4767-4769, Nov. 1994.

[3] M. Okano, K. Tsurumoto, S. Togo, N. Tamada, and S. Fuchino, "Characteristics of the magnetic gear using a bulk high-Tc superconductor," IEEE Trans. Appl. Supercond., vol. 12, no. 1, pp. 979-983, Mar. 2002.

[4] K. Atallah, S. D. Calverley, and D. Howe, "Design, analysis and realisation of a high-performance magnetic gear," IEE Proc.-Electric Power Appl., vol. 151, no. 2, pp. 135-143, Mar. 2004.

[5] K. T. Chau, D. Zhang, J. Z. Jiang, and L. N. Jian, "Transient analysis of coaxial magnetic gears using finite element comodeling," Journal of Applied Physics, vol. 103, no. 7, pp. 07F101:1-07F101:3, Jan. 2008.

[6] L. Jian and K. T. Chau, "Analytical calculation of magnetic field distribution in coaxial magnetic gears," Progress in Electromagnetics Research, vol. 92, pp. 1-16, 2009.

[7] K. T. Chau, D. Zhang, J. Z. Jiang, C. Liu, and Y. J. Zhang, "Design of a magnetic-geared outer-rotor permanent-magnet brushless motor for electric vehicles," IEEE Trans. Mag., vol. 43, no. 6, pp. 2504-2506, Jun. 2007.

[8] L. Jian, K. T. Chau, and J. Z. Jiang, "A magnetic-geared outer-rotor permanent-magnet brushless machine for wind power generation," IEEE Trans. Ind. Appli., vol. 45, no. 3, May/Jun. 2009.

[9] L. Jian, K. T. Chau, Y. Gong, J. Z. Jiang, C. Yu, and W. Li, "Comparison of coaxial magnetic gears with different topologies," IEEE Trans. Mag., vol. 45, no. 10, pp. 4526-4529, Oct. 2009.

[10] L. Jian and K. T. Chau, "A coaxial magnetic gear with Halbach permanent magnet arrays," IEEE Trans. Energy Conv., in press. 